\title{
ABSORCIÓN DE POTASIO MARCADO CON Rb-85 EN DIFERENTES SISTEMAS DE MANTENIMIENTO EN LA CORONA DE PALMA ACEITERA (Elaeis guineensis Jacq.).
}

\author{
Autores: \\ Marcelo Calvache Ulloa'; Francisco Recalde ${ }^{2}$
}

'Universidad Tecnológica Equinoccial. Campus Santo Domingo.

\section{REVISTA DE INVESTIGACIÓN CIENTIFICA}





\section{RESUMEN}

En el Centro de Investigaciones en Palma Aceitera (Cipal) de la Asociación Nacional de Cultivadores de Palma Africana (Ancupa), ubicado en La Concordia, a 260 m.s.n.m.; se estudió la absorción del potasio marcado con rubidio, bajo la influencia de diferentes sistemas de mantenimiento en la corona de palma aceitera, para lo cual se empleó un Diseño de Bloques Completos al Azar con cuatro repeticiones y 6 tratamientos. Se evaluaron las siguientes variables: contenido de potasio en la hoja $n^{\circ} 9$, contenido de rubidio en la hoja $\mathrm{n}^{\circ}$ 9, potasio proveniente del fertilizante y costos de los tratamientos en estudio. Con base en los resultados obtenidos se pudo concluir que el mejor sistema de mantenimiento fue el de corona cubierta con raquis. Las plantas que tenían la corona bajo raquis mostraron el mejor comportamiento con diferencias estadísticas en las variables: contenido de potasio en la hoja $\mathrm{n}^{\circ}$ 9, contenido de rubidio en la hoja $\mathrm{n}^{\circ} 9$, potasio derivado del fertilizante.

Descriptores: Absorción de potasio, actividad radicular, Palma aceitera (Elaeis guineensis Jacq.), rubidio-85, sistemas de mantenimiento de coronas.

\section{ABSTRACT}

In the Research Center of Oil Palm (Cipal) belonging to National Growers Association of Oil Palm (Ancupa), located in La Concordia area that is to 260 m.s.l.; the potassium absorption was tracer with rubidium, under the influence of different maintaining systems of oil palm circles, so that it was used Randomized complete blocks design with 4 replications and 6 treatments. It was evaluated the following variables: potassium contained in the leaf $n^{\circ} 9$, rubidium contained in the in the leaf $n^{\circ} 9$, potassium coming from the fertilizer, and the cost of studies treatment. Base on the obtained results it could conclude that the maintaining systems that had a direct effect on the potassium absorption was under empty fruit bunches, since it showed statistical differences in the variable: potassium contained in the leaf $n^{\circ} 9$, rubidium contained in the leaf $n^{\circ} 9$, potassium coming from the fertilizer.

Key words: Rubidium 85, potassium absorption and root activity, Oil Palm (Elaeis guineensis Jacq.), palm circles maintaining systems. 


\section{INTRODUCCIÓN}

La palma aceitera (Elaeis guineensis Jacq.) es importante en el Ecuador por su extensión de cultivo (207 225 ha), por el impacto social al generar puestos de trabajo en diferentes niveles desde jornaleros hasta personal especializado. Así, áreas que eran extremadamente olvidadas por las autoridades, ahora cuentan con centros de salud, educación y de alguna u otra manera ya son mejor atendidas. De la palma aceitera se obtienen productos comestibles y no comestibles como el biodiesel (Ancupa, 2006). A pesar de la importancia del cultivo, el rendimiento es bajo (2. 2 TM de aceite/ha) (Ancupa, 2006). Una de las causas para este bajo rendimiento es el manejo inapropiado del cultivo y dentro del manejo están las prácticas irregulares de fertilización. Otra práctica común es el mantenimiento de la "corona" para lograr mejores cosechas del fruto. Sin embargo, la limpieza con machete o con químicos puede afectar seriamente el sistema radicular y, por lo tanto, la absorción de los nutrientes, especialmente los macronutrientes como el potasio. Estas prácticas, de alguna manera, estarían contribuyendo con los bajos rendimientos.

Según Muter (1998), una función importante del potasio es la influencia en el uso del agua, especialmente en la tasa de transpiración. La apertura y cierre de los estomas es regulada por la concentración de $\mathrm{K}$ en las células que rodean los estomas El potasio $(\mathrm{K})$ es requerido para la síntesis proteica en mayores concentraciones que para la activación enzimática,

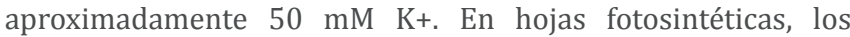
cloroplastos originan cerca de la mitad tanto de ácido ribunocleico foliar como de proteína foliar. En plantas C3 la mayoría de proteína cloroplástica es la ribulosabifosfato carboxilasa. Por consiguiente, se deteriora particularmente la síntesis de esta enzima bajo deficiencia de $\mathrm{K}$ y responde rápidamente al resuministro de $\mathrm{K}+$ [Marschner 1995].

Calvache (1999), señala que el potasio es el nutriente más absorbido por el cultivo de palma, exportándose en una cosecha de 25 t de fruta la cantidad de $94 \mathrm{~kg}$ de K. Además, la absorción total de la planta (material vegetativo, hojas podadas, racimos, inflorescencias) llega a $250 \mathrm{~kg}$ de $\mathrm{K} / \mathrm{año}$. El $\mathrm{K}$ participa en el desarrollo de los tejidos meristemáticos y juega un papel importante en el control del agua en la planta y en los procesos de evapotranspiración. Ante este escenario se plantearon los siguientes objetivos: Evaluar la influencia de diferentes sistemas de mantenimiento de la "corona" de palma aceitera (Elaeis guineensis Jacq.) en la absorción del potasio trazado con rubidio-85 (85Rb). Determinar el sistema de mantenimiento de la "corona" que permita una mejor absorción del potasio trazado con rubidio-85 $(85 \mathrm{Rb})$ en el cultivo de palma aceitera (Elaeis guineensis Jacq.).

\section{MÉTODOS Y MATERIALES}

La investigación se realizó en el Centro de Investigaciones en Palma Aceitera, Cipal, perteneciente a Asociación Nacional de Cultivadores de Palma Aceitera Ancupa, a $260 \mathrm{msnm}$, con una temperatura promedio de $24.2^{\circ} \mathrm{C}$ y una precipitación de 3071 $\mathrm{mm}$ al año. Se utilizaron plantas de palma aceitera del material Tenera Iniap. El factor en estudio fue: sistemas de mantenimiento de la "corona" y los tratamientos en estudio fueron: $\mathrm{t} 1$ : "corona" a machete, $\mathrm{t} 2$ : "corona" a machete + enraizador, $\mathrm{t} 3$ : "corona" + herbicida glifosato, t4: "corona" invertida, t5: "corona” cubierta con raquis, t6: "corona" cubierta con saquillos de polietileno.

Se utilizó un diseño de bloques completos al azar con cuatro repeticiones, que se tomaron respetando homogeneidad entre material vegetativo de palma aceitera (Elaeis guineensis Jacq.). La Unidad Experimental de $81 \mathrm{~m} 2$ estuvo formada por una planta de Palma aceitera (Elaeis guineensis Jacq.). Las variables evaluadas fueron: contenido de potasio en la hoja $\mathrm{n}$ - 9, contenido de rubidio en la hoja $n^{0}$ 9, potasio proveniente del fertilizante, emisión foliar, rendimiento total, incremento en la altura de planta, incremento del área foliar, peso fresco y peso seco total de malas hierbas.

Se aplicó al suelo sulfato de potasio marcado con sulfato de rubidio con 5,6 \% de átomos de $\mathrm{Rb}-85$, a los 7 meses de haber instalado los diferentes sistemas de mantenimiento de la "corona" de la palma aceitera, posteriormente al mes y a los dos meses de la aplicación de la dilución isotópica, se tomó muestras foliares a fin de analizar potasio y rubidio en el laboratorio. Para determinar la concentración de $\mathrm{K}$ y $\mathrm{Rb}$, se usó el método de «análisis foliar por el procedimiento de digestión vía húmeda con mezcla de ácido nítrico y perclórico». . Los cálculos isotópicos y del potasio proveniente del fertilizante se realizaron con la fórmulas de Calvache y Lopez [2000].

\section{RESULTADOS Y DISCUSIÓN}

\section{Contenido de potasio en la hoja no 9}

Del análisis de varianza para contenido de potasio en la hoja $\mathrm{n}^{\circ} 9$, al mes de aplicado el rubidio-85 (Tabla 1) se obtuvo un promedio de $0.87 \%$ y un coeficiente de variación de $8.20 \%$. Se observó que hay diferencias altamente significativas para sistemas, para las comparaciones ortogonales: s1, s2 vs. s3, s4, s5, s6; s1 vs. s2; s5 vs. s6, y no significación estadística para el resto de comparaciones ortogonales y bloques o repeticiones. Del análisis de varianza para contenido de potasio en la hoja ${ }^{\circ}$ 9, a los 2 meses de aplicado el rubidio (Tabla 1) se obtuvo un promedio de $0.61 \%$ y un coeficiente de variación de $13.23 \%$. Se observó que hay diferencias significativas para sistemas, para la cuarta comparación ortogonal: s4 vs. s5, s6, y no significación estadística para el resto de comparaciones ortogonales y bloques o repeticiones. 
Según Calvache (1999) el nivel adecuado de potasio de la hoja $n^{\circ} 9$ estaría entre 1.00 a $1.50 \%$. Sin embargo, los promedios obtenidos al mes y a los 2 meses de aplicado el rubidio $(0.87 \%)$ y $(0.61 \%)$ respectivamente son bajos y con tendencia decreciente. Probablemente, la planta reemplazó el potasio por el rubidio (Tabla 1).

Tabla 1. ADEVA general, en el estudio de seis sistemas de mantenimiento de la corona de palma aceitera (Elaeis guineensis Jacq.) sobre la absorción del potasio. La Concordia.

\begin{tabular}{|c|c|c|c|c|c|c|c|}
\hline \multirow{3}{*}{$\begin{array}{l}\text { FUENTE DE } \\
\text { VARIACIÓN }\end{array}$} & \multirow{3}{*}{ GL } & \multicolumn{6}{|c|}{ CUADRADOS MEDIOS } \\
\hline & & \multicolumn{3}{|c|}{$1 \mathrm{er} \mathrm{mes}^{1}$} & \multicolumn{3}{|c|}{2 do $\mathrm{mes}^{2}$} \\
\hline & & Potasio (\%) & Rubidio (ppm) & KddF (\%) & Potasio (\%) & Rubidio (ppm) & KddF (\%) \\
\hline Total & 23 & & & & & & \\
\hline Sistemas (S) & 5 & $0.08 * *$ & $360.74 * *$ & 0.89 ** & $0.02 *$ & 256.98 ** & $1.33^{* *}$ \\
\hline $\begin{array}{l}\text { s1, s2 vs. s3, s4, } \\
\text { s5, s6 }\end{array}$ & 1 & $0.17^{* *}$ & $355.98 * *$ & $0.10 \mathrm{~ns}$ & $0.01 \mathrm{~ns}$ & $148.79 *$ & $0.71^{\text {ns }}$ \\
\hline s1 vs. s2 & 1 & 0.10 ** & $6.35 \mathrm{~ns}$ & 0.58 ** & $0.03^{\mathrm{ns}}$ & $4.42^{\mathrm{ns}}$ & 0.04 \\
\hline s3 vs. s4, s5, s6 & 1 & $0.01^{\mathrm{ns}}$ & $138.61 * *$ & $0.99^{* *}$ & $0.01^{\mathrm{ns}}$ & $218.21^{* *}$ & $0.51^{\mathrm{ns}}$ \\
\hline s4 vs. s5, s6 & 1 & $0.02^{\mathrm{ns}}$ & $1.04^{\mathrm{ns}}$ & $0.03 \mathrm{~ns}$ & $0.03 *$ & $1.18 \mathrm{~ns}$ & $1.32 *$ \\
\hline s5 vs. s6 & 1 & $0.08^{* *}$ & 1301.72 ** & 2.75 ** & $0.02^{\text {ns }}$ & 912.30 ** & $4.10^{* *}$ \\
\hline Repeticiones & 3 & $0.01^{\mathrm{ns}}$ & $0.81^{\mathrm{ns}}$ & $0.02^{\mathrm{ns}}$ & $0.00^{\mathrm{ns}}$ & $33.65 \mathrm{~ns}$ & $0.84 *$ \\
\hline Error Exp. & 15 & 0.01 & 8.92 & 0.05 & 0.01 & 21.63 & 0.23 \\
\hline Promedio & & 0.87 & 29.17 & 0.78 & 0.61 & 34.29 & 2.02 \\
\hline CV (\%) & & 8.20 & 10.24 & 28.61 & 13.23 & 13.56 & 23.60 \\
\hline
\end{tabular}

Potasio al mes de aplicado la dilución de sulfato de potasio más sulfato de rubidio

Al aplicar Tukey al 5\% para contenido de potasio en la hoja $\mathrm{n}^{\circ}$ 9, bajo la influencia de los sistemas (Tabla 2) se observaron, 3 rangos de significación estadística. Encabezando el primer rango estuvieron los sistemas (s3, s4 y s5), siendo el sistema s5 (Raquis), el que presentó el mayor promedio con 1.02\%. En el segundo rango se mantuvieron los sistemas (s2 y s6), y finalmente en el último rango se mantuvo el sistema s1 (Machete) con un promedio de $0.63 \%$.

Tabla 2. Promedio de potasio en la hoja $\mathrm{n}^{\circ} 9$, al mes de aplicado al suelo, en el estudio de seis sistemas de mantenimiento de la corona de palma aceitera (Elaeis guineensis Jacq.) sobre la absorción del potasio. La Concordia.

\begin{tabular}{c|cc}
\multicolumn{2}{c|}{ SISTEMAS DE MANTENIMIENTO } & \multicolumn{2}{c}{ POTASIO (\%) } \\
Codificación & Significado & \\
\hline s5 & Raquis & $0.99 \mathrm{a}$ \\
s4 & Corona invertida & $0.89 \mathrm{a}$ \\
\hline s3 & Glifosato & $0.86 \mathrm{ab}$ \\
\hline s2 & Machete más enraizador & $0.81 \mathrm{~b}$ \\
\hline s6 & Saquillos & $0.63 \mathrm{c}$ \\
\hline
\end{tabular}

Al encontrar diferencias significativas al mes y a los dos meses (Calvache, 2008).

\section{Contenido de rubidio en la hoja no 9} de aplicado el rubidio sobre el contenido de potasio en la hoja ${ }^{\circ}$ 9, bajo el sistema s1 (Machete) que ha sido el más común en las zonas donde se cultiva palma aceitera, se podría decir que este sistema es uno más de los factores que conllevaría a incrementar el problema de desbalance nutricional entre potasio, calcio y magnesio, tan común en varias zonas palmicultoras del país

Del análisis de varianza para rubidio en la hoja $\mathrm{n}^{\circ} 9$ al primer mes de aplicado el rubidio (Tabla 1) se obtuvo un promedio de

Potasio a los 2 meses de aplicado la dilución de sulfato de potasio más sulfato de rubidio

influencia de los sistemas (Tabla 3), se observaron, 2 rangos de significación estadística. Encabezando el primer rango se mantuvo el sistema s5 (Raquis) con $0.71 \%$, y en el segundo

Tabla 3. Promedio de potasio en la hoja $n^{\circ} 9$, a los 2 meses de aplicado al suelo, en el estudio de seis sistemas de mantenimiento absorción del potasio. La Concordia.

\begin{tabular}{c|ccc}
\multicolumn{2}{c}{ SISTEMAS DE MANTENIMIENTO } & \multicolumn{2}{c}{ POTASIO (\%) } \\
\hline Codificación & Significado & & $\mathrm{a}$ \\
\hline s5 & Raquis & 0.71 & $\mathrm{ab}$ \\
\hline s3 & Glifosato & 0.68 & $\mathrm{ab}$ \\
\hline s2 & Machete más enraizador & 0.64 & $\mathrm{ab}$ \\
\hline s6 & Saquillos & 0.60 & $\mathrm{ab}$ \\
s4 1 & Corona invertida & 0.54 & $\mathrm{~b}$ \\
\end{tabular}

Según Hartley (1983) las raíces de palma muestran un crecimiento positivo hacia las mejores condiciones de de palma en descomposición, esto puede conducir hacia una gran densidad de raíces cuaternarias. Entonces, al ser el sistema s5 (Raquis) una forma de materia orgánica luego de descomponerse, es de suponerse que se estaría mejorando la
absorción del potasio, aspecto que es apreciado en el porcentaje de potasio en la hoja $\mathrm{n}^{\circ} 9$, al mes y a los dos meses de aplicado el

Maldonado (1965), manifiesta que hay que cuidar de no causar daño a las raíces de palma que son bastante superficiales, pues comúnmente se las corta cuando se realiza la práctica llamada coro machete. Esta sería la rán por lo cual el sistema de potasio entre los sistemas en estudio al mes de aplicado el rubidio donde se registró diferencias estadísticas y por otro lado ocupó el segundo rango a los dos meses de la aplicación del rubidio (Tablas 2 y 3 ). 
29.17 ppm (partes por millón) y un coeficiente de variación de $10.24 \%$. Se observó que hay diferencias altamente significativas para sistemas, para las comparaciones ortogonales: s1, s2 vs. s3, s4, s5, s6; s3 vs. s4, s5, s6; s5 vs. s6, y no significación estadística para el resto de comparaciones ortogonales y bloques o repeticiones. Del análisis de varianza para rubidio en la hoja $\mathrm{n}^{\circ} 9$ a los 2 meses de aplicado rubidio (Tabla1) se obtuvo un promedio de 34.29 ppm y un coeficiente de variación de $13.56 \%$. Se observó que hay diferencias altamente significativas para sistemas, para las comparaciones ortogonales: s3 vs. s4, s5, s6; s5 vs. s6, diferencias significativas para la primera comparación ortogonal: s1, s2 vs. s3, s4, s5, s6, y no significación estadística para el resto de comparaciones ortogonales y bloques 0 repeticiones.

Según Tukey al 5\% para rubidio en la hoja $n^{\circ} 9$, al primer y segundo mes de aplicado al suelo, bajo la influencia de los sistemas (Tabla 4), se observaron 3 rangos de significación estadística. Encabezando el primer rango estuvieron los sistemas (s3 y s5) siendo el sistema s5 (Raquis), el mayor con un promedio de 43.16 y 44.38 ppm respectivamente, y en el último rango el sistema s6 (Saquillos) con 17.65 y 23.02 ppm respectivamente

Cerón (2004), manifiesta que la competencia reduce el vigor de las plantas cultivadas, debido a que las malezas merman el agua, nutrientes, luz y el CO2, tomándolo para su desarrollo y que al eliminarlas con herbicidas se elimina esta competencia. Esta sería la razón por la que el rubidio en la hoja $n^{\circ} 9$ al mes y a los 2 meses de aplicado el rubidio en el sistema s3 (Glifosato) se mantuvieron también en el primer rango entre los sistemas en estudio (Tabla 4).

Tabla 4. Promedio de rubidio en la hoja $n^{\circ} 9$, en el estudio de seis sistemas de mantenimiento de la corona de palma aceitera (Elaeis guineensis Jacq.) sobre la absorción del potasio. La Concordia.

\begin{tabular}{c|ccc}
\multicolumn{2}{c}{ SISTEMAS DE MANTENIMIENTO } & \multicolumn{2}{c}{ RUBIDIO (PPM) } \\
Codificación & Significado & 1er mes & 2do mes \\
\hline s5 & Raquis & $43.16 \mathrm{a}$ & $44.38 \mathrm{a}$ \\
s3 & Glifosato & $36.99 \mathrm{a}$ & $42.45 \mathrm{a}$ \\
\hline s4 & Corona invertida & $29.78 \mathrm{~b}$ & $34.36 \mathrm{ab}$ \\
s2 & Machete más enraizador & $24.61 \mathrm{bc}$ & $31.51 \mathrm{bc}$ \\
\hline s1 & Machete & $22.83 \mathrm{bc}$ & $30.03 \mathrm{bc}$ \\
s6 & Saquillos & $17.65 \mathrm{c}$ & $23.02 \mathrm{c}$ \\
\hline
\end{tabular}

\section{Potasio derivado del Fertilizante (KddF)}

Del análisis de varianza para KddF al mes de haber aplicado el rubidio (Tabla 1), se obtuvo un promedio de $0.78 \%$ y un coeficiente de variación de $28.61 \%$. Se observó que hay diferencias altamente significativas para sistemas, para las comparaciones ortogonales: s1 vs. s2; s3 vs. s4, s5, s6; s5 vs. s6, y no significación estadística para el resto de comparaciones ortogonales y bloques o repeticiones. Del análisis de varianza para KddF a los 2 meses de haber aplicado el rubidio (Tabla 1 ), se obtuvo un promedio de $2.02 \%$ y un coeficiente de variación de $23.60 \%$. Se observó que hay diferencias altamente significativas para sistemas, para la comparación ortogonal: s5 vs. s6, diferencias significativas para la cuarta comparación ortogonal: s4 vs. s5, s6 y no significación estadística para el resto de comparaciones ortogonales.

De acuerdo con los promedios obtenidos en la variable KddF (Tabla 1) que fueron de $0.78 \%$ y $2.02 \%$, son bajos en comparación con previas investigaciones (Calvache, 1999), donde en experimentos de distancias de aplicación y fraccionamiento obtuvo valores de $2.43 \%$ y $6.15 \%$ respectivamente. La explicación a estos resultados serían las abundantes lluvias que se presentaron, con precipitaciones acumuladas que alcanzaron el orden de los $963.5 \mathrm{~mm}$ al mes de la aplicación del rubidio y $327.5 \mathrm{~mm}$ desde el mes hasta los dos meses de la aplicación del rubidio, dando un total de $1291 \mathrm{~mm}$, cuando la palma aceitera necesita de 120 a $150 \mathrm{~mm} / \mathrm{mes}$, bien distribuidos (Ancupa, 2003) por lo cual es lógico que gran parte del isótopo aplicado se habría lavado justificando los resultados obtenidos.

\section{Potasio derivado del Fertilizante al mes de aplicado la} dilución de sulfato de potasio más sulfato de rubidio

Al aplicar Tukey al 5\% para KddF, bajo la influencia de los sistemas (Tabla 5) se observaron, 3 rangos de significación estadística, en el primer rango se mantuvieron los sistemas (s1, s3 y s5) siendo el sistema s5 (Raquis), el que mayor promedio presentó con $1.30 \%$. En el segundo rango se mantuvieron los sistemas (s1 y s4) y finalmente en el último rango se mantuvieron los sistemas (s2, s4 y s6), siendo el sistema s6 (Saquillos) el que promedio presentó $0.13 \%$.

Tabla 5. Promedio de potasio derivado del fertilizante, en el estudio de seis sistemas de mantenimiento de la corona de palma aceitera (Elaeis guineensis Jacq.) sobre la absorción del potasio al mes de aplicado. La Concordia.

\begin{tabular}{c|cc}
\multicolumn{2}{|c}{ SISTEMAS DE MANTENIMIENTO } & \multicolumn{2}{c}{ POTASIO PROVENIENTE } \\
Codificación & Significado & DEL FERTILIZANTE (\%) \\
\hline s5 & Raquis & $1.30 \mathrm{a}$ \\
s3 & Glifosato & $1.25 \mathrm{a}$ \\
\hline s1 & Machete & $0.96 \mathrm{ab}$ \\
\hline s4 & Corona invertida & $0.61 \mathrm{bc}$ \\
\hline s2 & Machete más enraizador & $0.42 \mathrm{c}$ \\
\hline s6 & Saquillos & $0.13 \mathrm{c}$ \\
\hline
\end{tabular}

Potasio derivado del Fertilizante a los 2 meses de aplicado la dilución de sulfato de potasio más sulfato de rubidio

Al aplicar Tukey al 5\% para KddF, bajo la influencia de los sistemas (Tabla 6), se observaron 2 rangos de significación estadística, en el primer rango estuvieron los sistemas (s1, s2, s3, s4 y s5) siendo el sistema s5 (Raquis), el que mayor 
promedio presentó con $2.52 \%$, en tanto que en el último rango se mantuvieron los sistemas (s1, s2 y s6) siendo el sistema s6 (Saquillos) el menor con 1.09\%.

Vallejo et al (1981), manifiestan que se ha encontrado un mayor número de raíces absorbentes en las zonas de mayor concentración de materia orgánica en descomposición, principalmente en las zonas donde se colocan las hojas después de la cosecha o poda y/o debajo de los troncos de madera en descomposición. El raquis, que luego del proceso de descomposición se transforma en materia orgánica, estaría favoreciendo la absorción del fertilizante, aplicado tal como lo sostienen los párrafos anteriores; y sería por esto que la variable potasio proveniente del fertilizante, bajo el sistema s5 (raquis) se encuentra encabezando el primer rango tanto al mes como a los dos meses de haber aplicado el rubidio-85 (Tablas 5 y 6).

Tabla 6. Promedio de potasio derivado del fertilizante, en el estudio de seis sistemas de mantenimiento de la corona de palma aceitera (Elaeis guineensis Jacq.) sobre la absorción del potasio, a los 2 meses de aplicado. La Concordia.

\begin{tabular}{|c|cc}
\hline \multicolumn{2}{|c}{ SISTEMAS DE MANTENIMIENTO } & $\begin{array}{c}\text { POTASIO PROVENIENTE DEL } \\
\text { FERTILIZANTE }(\%)\end{array}$ \\
\hline Codificación & Significado & $2.52 \mathrm{a}$ \\
\hline s5 & Raquis & $2.51 \mathrm{a}$ \\
\hline s4 3 & Corona invertida & $2.45 \mathrm{a}$ \\
s1 & Glifosato & $1.85 \mathrm{ab}$ \\
\hline s2 & Machete & $1.71 \mathrm{ab}$ \\
s6 & Machete más enraizador & $1.09 \mathrm{~b}$ \\
\hline
\end{tabular}

Cerón (2004), concuerda que una de las ventajas del uso de herbicidas es permitir un mayor aprovechamiento de los fertilizantes aplicados. Esta sería la explicación para los resultados obtenidos al mes de aplicado el rubidio, donde se observó que el potasio derivado del fertilizante en el sistema s3 (glifosato) se mantuvo en el primer rango (Tabla 6).

Según Dubois (1980), al ser el plástico impermeable al vapor de agua y a los líquidos impide la evaporación del agua del suelo, con el efecto consiguiente que se mantiene a la disposición de las plantas cultivadas. Conviene realizar sobre el filme pequeñas perforaciones para evitar que el agua se acumule sobre su superficie, pasando ésta al suelo para que pueda ser aprovechada por el árbol. Esto explicaría porque el sistema con saquillos presentó menores valores en absorción de rubidio y potasio proveniente del fertilizante (Tablas 4,5 , y 6), pues, en el caso del ensayo se colocó saquillos los mismos que no garantizaban una impermeabilización completa y uniforme ya que, cubrían toda el área de la "corona" pero por secciones diferenciadas y desiguales, dejando lugares que si bien estaban cubiertos por saquillos no garantizaban una impermeabilización contrario a filmes de plásticos propuestos por Dubois (1980). En el caso del ensayo, por un lado, la poca agua que había llegado a la rizósfera se podía evaporar y, por otro lado, el agua de la lluvia se quedaba sobre la superficie de los saquillos que se habían impermeabilizado por el polvo y tierra propios del área, teniendo como resultado que la rizósfera de la palma bajo los saquillos de polietileno estaba seca, a pesar de la lluvia y del sistema de riego.

El sistema s6 (Saquillos) ocupó el último rango en las variables: contenido de rubidio en la hoja $n^{\circ} 9$, potasio proveniente del fertilizante al mes y dos meses de haber aplicado el rubidio. Esto se debería a que los saquillos cubren en su totalidad el suelo impidiendo que el agua de riego por aspersión ingrese directamente a la rizósfera del cultivo y la mayor parte del tiempo, durante la investigación coincidió con la época seca, por lo que al levantar los saquillos el suelo estaba seco previo a la aplicación del rubidio. Sin embargo, hay que recalcar que comenzó a llover 1 mes antes de la aplicación del trazador y aparentemente este tiempo no fue suficiente para la recuperación del sistema radicular en los sistemas s6 (saquillos) (Tablas 4, 5 y 6).

\section{CONCLUSIONES}

- Los sistemas de mantenimiento de coronas de palma aceitera, s5 (corona cubierta con raquis) y s3 (corona con glifosato) tuvieron los mejores resultados sobre las siguientes variables evaluadas: potasio, rubidio presentes en la hoja $\mathrm{n}^{\circ} 9$, potasio proveniente del fertilizante.

\section{RECOMENDACIONES}

Bajo las condiciones agroecológicas específicas de la zona de La Concordia, se recomienda:

- Mantener el sistema de mantenimiento s5 (corona cubierta con raquis), como parte de los programas de manejo del cultivo de palma aceitera. Cuando exista invasión de malas yerbas aplicar glifosato en la corona

- Realizar otras investigaciones utilizando trazadores como: nitrógeno-15, fósforo-32 a fin de determinar la absorción real de estos elementos, bajo la influencia de los diferentes sistemas de mantenimiento en estudio. 


\section{REFERENCIAS BIBLIOGRÁFICAS}

1. Ancupa. 2003. Manual del cultivo de Palma aceitera. (Elaeis guineensis Jacq.). Quito. p 125

2. Ancupa. 2006. Manejo de nutrientes y fertilización de la fase inmadura. Asociación Nacional de Cultivadores de Palma Africana. Ecuador. No. 6. 14 - 15; 20 p

3. Calvache, M. 1999. Determinación de la eficiencia de la aplicación de potasio en el cultivo de palma africana utilizando 85Rb como trazador. Revista El palmicultor No. 13:13 - 15

4. Calvache, M. 2008. Fertirrigación en palma aceitera. Memorias del Seminario Internacional Nutrición y Manejo de Palma Aceitera. IPNI-SECS.CD

5. Calvache, M., y M. López. 2000. Evaluación de la eficiencia de la fertilización potásica en el cultivo de palma africana utilizando 85Rb como trazador. Informaciones agronómicas 38:8-10.

6. Cerón, J. 2004. Evaluación de mezclas de herbicidas y una siloxanona en el cultivo de arroz (Oryza sativa L.) La Troncal Cañar. Tesis Ing. Agrónomo. Universidad Central del Ecuador. Facultad de Ciencias Agrícolas. Quito p 18,19; 134 p.

7. Dubois, P. 1980. Los plásticos en la Agricultura. Trad. por Brighton. Editorial Mundi-Prensa. Madrid (España). 209 Hartley, 1983. La palma de aceite (Elaeis guineensis Jacq.), segunda edición. México. p. 15 - 138, 210 - 225

8. Hartley, m. 1983 La palma de aceite Trad. Maldonado. 2 ed. México, ME

9. Maldonado, E. 1965. La palma africana o palma de aceite. Instituto Nacional de Investigaciones Agropecuarias Santo Domingo. Quito (Ecuador) $40 \mathrm{p}$

10. Marschner, H. 1995. Mineral nutrition of higher plants, 2 ed., Hohenheim.

11. Mutert, E. 1998. El potasio en la palma aceitera. Quito, EC. Revista El Palmicultor 12:6-11

12. Vallejo, G.; Figueredo, P.; Rojas, L.; Muñoz, R.1981. La palma africana de aceite. 149 Ed. Bogotá (Colombia). p 17 - 22; 212 